\title{
Deux nouvelles lettres de Saint-Just à Garot
}

\section{Bernard Vinot}

\section{(2) OpenEdition \\ Journals}

Édition électronique

URL : https://journals.openedition.org/ahrf/7913

DOI : 10.4000/ahrf.7913

ISSN : 1952-403X

Éditeur :

Armand Colin, Société des études robespierristes

Édition imprimée

Date de publication : 1 décembre 2006

Pagination : 133-143

ISSN : 0003-4436

\section{Référence électronique}

Bernard Vinot, «Deux nouvelles lettres de Saint-Just à Garot ». Annales historiques de la Révolution

française [En ligne], 346 | Octobre/Décembre 2006, mis en ligne le 01 décembre 2009, consulté le 28 juin 2022. URL : http://journals.openedition.org/ahrf/7913 ; DOI : https://doi.org/10.4000/ahrf.7913

Ce document a été généré automatiquement le 24 avril 2022.

Tous droits réservés 


\title{
Deux nouvelles lettres de Saint-Just à Garot
}

\author{
Bernard Vinot
}

1 On ne connaissait pas la première de ces deux lettres, sortie d'une collection privée et offerte à la vente publique à la fin de l'année $2004^{1}$. En voici le texte intégral ${ }^{2}$ :

« Je vous envoie Monsieur et ami une affaire assez particulière. Comme on m'a dit qu'il y avait audience le vendredi et que notre partie est citée pour la prochaine audience, j'ai pris la liberté de faire des moyens que vous restreindrez ou étendrez comme il vous plaira. J'ai bien prévu que vous n'auriez pas grand temps avant cette audience, c'est pourquoi j'ai pris les devants.

Vous verrez qu'il s'agit d'un homme qui n'entendant être que caution s'est laissé comprendre dans le contrat sous le titre d'acquéreur pour d'autres, et j'ai entrepris de restreindre cette dernière qualité aux termes de droit. Me Gellé [sera] encore votre partie adverse.

Je vous prie de me répondre par la plus prochaine occasion à la question suivante qui concerne une autre affaire.

Mr...... a cité au bureau de paix de Blérancourt le possesseur de quelques propres maternels qu'il prétend revendiquer. Le possesseur et les biens sont dans le canton. Le possesseur cité comme je l'espérais a dénoncé ses bailleurs. Ils demeurent à Compiègne. Donnez-moi un chemin pour mener cette affaire au tribunal de Couci au lieu d'aller au tribunal des défendeurs à Compiègne.

Autre affaire. Le sieur Pigny et le Pr [procureur] de la commune ont été interpellés à l'audience. Faites savoir le plus tôt possible. Je crois que le sieur Pigny n'a point fait d'offres du tout, mais il est très certain qu'il n'en a point fait d'écrites. Il faudrait que personne ne perde, mais j'aime encore mieux qu'un seul succombe.

La sentence afin de dépens pour les dragons a été signifiée aux officiers municipaux par expt [exploit] de Gibault ${ }^{3}$. Le même Gibault le même jour de cette signification a saisi toutes les redevances de la commune sans autre délai. Le lendemain le rôle de répartition a été fait. Conséquemment les ss. Pigny et Massi sont responsables de leur impatience et de l'illégalité de leurs saisies. [verso] D'autant plus que dans l'assemblée convoquée pour signer le rôle et le rendre exécutoire, le même s. Pigny qui avait fait saisir s'opposa à l'exécution du rôle, ce qui sera déduit s'il en est besoin.

2do [Secondo] Remarquez bien que la sentence n'était qu'à fin de dépens et que comme elle ne [préjudiciait rayé] décidait $\mathrm{ni}^{4}$ ne pouvait rien décider sur le fond, les 
ss. Pigny et Massi ne pouvaient point saisir pour les dépens, mais bien vous qui étiez le procureur. Faites entendre cela au sieur Pigny et dites-lui que tout cela sera hardiment soutenu et qu'il prenne des sûretés prudemment.

Les ss. Pigny et Massi auraient bien pu soutenir non point en vertu de la susdite sentence mais en vertu de l'arrêté du département. Il faut à toutes choses son mode, ses convenances.

Ne parlez aucunement de moi à ces gens-là. C'est un salutaire avis que je leur donne par votre bouche. Je voudrais que tous les hommes fussent sages et d'un bon esprit. Répondez-moi au plus tôt. Demandez la délivrance des des [sic] consignés dans l'affaire de Mr Daubigni afin que le sieur Lesassière et l'auteur soient payés sans plus de frais.

Je vous embrasse ainsi que votre épouse que je prie d'agréer mon respect et suis tout à vous.

Saint-Just

Faites vite juger ce qui regarde Mr Decaisne. Mes compliments à Mr Hottin.

La lettre est adressée à : Monsieur Garot avoué

Près le tribunal de Couci

Et l'on peut lire en-dessous de l'adresse: "après avoir fermé ma lettre [je m'aperçois que] les pièces de Louis Honnoré contre Mr Gellé pour vente d'une maison sise à Blérancourt ne sont point entre mes mains et Mr Decaisne est absent. Parler à Mr Bottée procureur pour Mr Gellé et demander huitaine ».

Charles Jean François Garot est un des correspondants réguliers de Saint-Just. Les deux hommes sont de générations différentes mais de même milieu puisque Garot est né en 1750 dans une famille de fermiers aisés au village de Juvigny implanté sur les riches terres du plateau soissonnais. Il est passé de la terre à l'office en reprenant l'étude de Me Canivet à Coucy-le-Château où il exerce à la fois les fonctions de notaire et de procureur près le tribunal de bailliage, puis de district. On ne sait pas comment SaintJust, de dix-sept ans son cadet, est entré en relation avec lui, mais peut-être par l'intermédiaire de ses cousins Lemoine ${ }^{5}$, fermiers de la grosse ferme de Loire et euxmêmes proches du notaire de Coucy. De toutes façons les affinités sont évidentes: Garot lui aussi s'est engagé dans les forces de rénovation dès avant la Révolution. Il passe à l'Intendance de Soissons dès 1788 pour un trublion ${ }^{6}$ et dans le cahier de doléances de son village natal qu'il a signé et probablement co-rédigé, on recommande une limitation de la taille des fermes à trois charrues pour ne pas ôter «le pain à un père de famille en lui prenant son emploi ${ }^{7}$. Autant que l'étroitesse des sources disponibles permette d'en juger, les relations des deux hommes resteront chaleureuses. Maire de Coucy-le-Château en 1793, Garot sera dénoncé par ses adversaires après Thermidor comme un fidèle de Saint-Just. ${ }^{8}$ Pas étonnant donc que cette connivence d'opinion aboutisse à des échanges le plus souvent de nature professionnelle ainsi qu'en témoigne cette nouvelle lettre inédite.

3 Sur le fond, elle confirme le cercle des amis, des adversaires et le degré de compétences juridiques de l'auteur qui se comporte moins en client qu'en jeune collègue demandant conseil mais n'hésitant pas également, en proposant, certes avec tact, ses propres moyens, à s'ériger en conseiller de son procureur. Au nombre des relations, on relève sans surprise les noms connus par ailleurs de Marie Louis Grégoire Hottin', probablement proche de Garot qui est chargé de transmettre les « compliments » dans cette lettre comme dans celle d'octobre $1790^{10}$, de Louis Honnoré, premier maire de la nouvelle municipalité de Blérancourt, du beau-frère Decaisne et du fort peu recommandable Daubigny. Mentor de Saint-Just à cette époque, celui-ci se répandra en dénigrements sur son compte après Thermidor. C'est probablement lui qui a livré avec retard à Courtois la fameuse lettre dont il était le destinataire, soi-disant retrouvée 
dans les papiers du Conventionnel exécuté, mais ne figurant pas dans la première édition du rapport de nivôse an III $^{11}$.

4 Les allusions aux affaires évoquées ne bouleversent donc pas ce que l'on sait par ailleurs du compagnonnage de Saint-Just à Blérancourt, mais le commentaire sur l'affaire Pigny vient éclairer l'incident de la Saint-Nicolas 1789 au cours duquel les partisans de Saint-Just avaient imposé une vente à bas prix des grains sur le marché de Blérancourt ${ }^{12}$. Le clan du Château avait exploité cette maladresse, politiquement très contre-productive, pour reprendre l'initiative dans l'assemblée communale. Plusieurs de ses délibérations recommandaient de faire appel à la troupe basée à Noyon pour assurer l'ordre sur les marchés sans qu'on sache si ces menaces avaient été suivies d'effet. La lettre confirme que des « dragons » sont effectivement venus aux frais de la commune de Blérancourt et qu'ils ont été accueillis par les aubergistes Pigny et Massy, affidés de la seigneurie. C'est la note de frais présentée à la nouvelle municipalité et impayée qui fait l'objet d'un litige au tribunal de Coucy ${ }^{13}$. La datation peut être évaluée avec précision grâce à la consultation du registre des délibérations communales de Blérancourt précisant que l'exploit de Gibault dont il est question dans la lettre a été signifié au procureur de la commune le lundi 21 juin $1790^{14}$. Saint-Just écrit donc le lendemain ou le surlendemain en prévision de l'audience du vendredi suivant, au plus tard la semaine d'après, ce qui, dans la correspondance Saint-Just Garot, permet de classer chronologiquement cette lettre en première position de celles qui nous sont parvenues jusqu'alors.

5 En complément de l'affaire Pigny et Massy, le même registre de délibérations donne une idée assez précise de la façon dont Saint-Just préparait le travail pour Garot. Voici en effet les moyens, écrits de la main de Thuillier, qu'oppose le conseil général de la commune aux prétentions des deux aubergistes le 22 juin 1790 :

« $1^{\circ}$ Comme nous ne pouvons souscrire à aucun engagement sans autorisation et que pour l'obtenir il faut une délibération de la commune, nous renvoyons à dimanche prochain l'assemblée générale de la paroisse et lui présenterons les mémoires des sieurs Pigny et Massy pour frais de troupes.

$2^{\circ}$ La municipalité nouvelle est-elle comptable des engagements pris par l'ancienne municipalité sans autorisation de l'Intendant ou de la commission provinciale alors en vigueur?

$3^{\circ}$ Le S. Pigny, en particulier dans un mémoire adressé à M. le maire, tendant à l'acquittement de son mémoire a avancé qu'il avait fourni des vivres en vertu d'un ordre ministériel et $\mathrm{M}$. le maire lui ayant par écrit demandé communication de cet ordre, le S. Pigny n'a plus parlé de rien.

$4^{\circ}$ Nous avons fouillé dans les archives de la municipalité et n'y avons trouvé aucune soumission faite par la commune à l'entretien des troupes soldées, ni aucune autorisation quelconque.

$5^{\circ} \mathrm{A}-\mathrm{t}-\mathrm{on}$ le droit de nous demander compte d'un engagement prétendu et secret auquel nous n'avons point eu de part et dans un temps où la troupe placée dans les marchés ne pouvait favoriser que la perception des droits seigneuriaux étrangers à la communauté ? à qui appartenait le marché ? au seigneur puisqu'il en affermait les droits 1500 à 2000 livres.

$6^{\circ}$ Dans tous les cas doit-on allouer de confiance aux dits Pigny et Massy ce qu'ils demandent? Quelle est la paye extraordinaire que mérite un cavalier? lui doit-on trois livres et plus par jour?

$7^{\circ}$ La paroisse s'est opposée formellement à une garde étrangère. Elle a fait ellemême le service avec trente hommes chaque jour de marché depuis le 14 décembre dernier. Différents arrêtés signés de tous les citoyens et déposés en original dans les mains de la commission intermédiaire de Soissons attestent sa bonne volonté pour 
l'ordre public.

$8^{\circ}$ En outre, n'étant assigné en vertu d'aucun titre d'aucune commission mais seulement en vertu d'une dépense arbitrairement faite, étant sans caractère à l'époque dont argumentent les S. Pigny et Massy, notre ministère n'étant point rétroactif, et le soin de l'acquittement ne nous étant transmis par aucun acte, nous ne devons ni ne pouvons les croire sur leur parole.

Nous demandons que les sieurs Pigny et Massy soient tenus de justifier de leur demande par devant le directoire de district et de département seuls compétents en matière d'engagement de contributions des communautés, que les dits sieurs soient condamnés aux dépens et qu'il soit donné à Monneveux, procureur de la commune, porteur des présentes expéditions, acte de son déplacement $»$.

Cette citation un peu longue mais qui mériterait de figurer dans les cuvres complètes ne néglige, comme on le voit, aucun argument juridique, historique et même politique. Bien des professionnels expérimentés du barreau pourraient la revendiquer.

7 La seconde lettre, conservée depuis longtemps à la bibliothèque municipale de Laon ${ }^{15}$, n'a été publiée qu'en $2004^{16}$ sans commentaire et présentée comme une correspondance à l'intention d'un «destinataire inconnu ». Son existence n'était pas ignorée, mais elle n'avait jamais retenu l'attention des chercheurs, peut-être parce qu'ils l'ont estimée d'un intérêt limité, sans doute à cause des difficultés d'interprétation qu'elle présente. Qu'on en juge :

« Paris le 8 juillet

Citoyen et ami,

Il y a quelque temps que je ne vous ai donné de mes nouvelles, je n'avais rien oublié cependant des témoignages d'amitié que vous m'avez donnés. N'aurai-je point le plaisir de vous revoir ici à la même époque où nous nous y sommes vus l'an passé ?

J'ai peu de loisir, je fais ce que je puis pour répondre à votre confiance et pourvu que je rende compte au peuple de mes moments, l'amitié ne sera pas plus sévère. Je vais m'occuper avec le citoyen Chassaie ${ }^{17}$ de l'affaire du citoyen Bailli que je vous prie d'assurer de mon sincère attachement ${ }^{18}$.

Dites s'il vous plaît à nos connaissances communes que je ne les ai point oubliées.

J'embrasse votre épouse, vos enfants et vous.

Saint-Just »

8 L'interprétation de cette lettre sans adresse et avec une date ne précisant pas l'année est plus épineuse. Le destinataire est à l'évidence une personnalité qui s'intègre dans le cercle des "connaissances communes» du pays d'origine avec laquelle Saint-Just maintient des échanges suivis, seulement interrompus par la charge du travail quotidien. Il l'aborde avec reconnaissance et une déférente amitié. La comparaison avec les lettres à Garot connues est édifiante ${ }^{19}$. Elles commencent toutes par "Monsieur et ami », ici «Citoyen et ami » : époque oblige! Elles incluent presque toutes « Madame» dans la formule de politesse, ici madame et les enfants (les Garot ont alors un fils et deux filles). Le ton est conforme à celui qu'emploieraient deux familles qui se connaissent bien. Le contrat de mariage Emmanuel Decaisne-Louise Saint-Just a été rédigé à l'étude Garot et c'est chez les Garot à Coucy qu'a été hébergé Saint-Just à l'occasion de l'élection des juges du district ${ }^{20}$, ce qui est conforme tant au témoignage d'amitié dont il est question ici qu'à la visite à Paris qu'aurait rendue l'année précédente le correspondant inconnu. On suppose enfin que celui-ci a pu s'entremettre pour solliciter l'appui du conventionnel en faveur d'un certain « citoyen Bailli ». Cette précieuse allusion oriente l'enquête vers l'intimité familiale des Garot. On découvre vite qu'à la naissance de leur fille Geneviève Olympe, le 2 avril 1793, l'enfant est présentée aux autorités par le père "assisté de Jean-Baptiste Lemoine ${ }^{21}$, 32 ans, demeurant à la 
ferme de Loire, commune de Troli et de Geneviève Courtenay, 69 ans, femme de Charles Maximin Bailli, laboureur demeurant à Malhôtel, commune de Crécy-au-mont $»^{22}$. Voici donc les Bailly, les Lemoine et les Garot alliés dans le même acte de sociabilité. Mais qui sont les Bailly et que veulent-ils qui soit de nature à nécessiter l'intervention d'une personnalité politique en vue comme Saint-Just?

Localement, Charles Maximin Bailly est loin d'être un inconnu. Fils d'un procureur d'Erquinvillers dans le Beauvaisis, il n'est arrivé à Crécy-au-Mont ${ }^{23}$ qu'à l'âge de 22 ans en 1759 pour épouser un an plus tard Geneviève Courtenay, veuve Pottier, de quatorze ans son aînée, qui exploitait deux belles fermes de la région : celle de Malhôtel, près de 200 jalois ${ }^{24}$ louée à la mense abbatiale de Nogent-sous-Coucy et celle des Tournelles, 400 arpents, propriété du sieur Le Pelletier, ancien capitaine d'artillerie et seigneur de Crécy-au-Mont. Avec 2053 livres d'impositions ${ }^{25}$ à la veille de la Révolution, Bailly est le plus gros contribuable de la région. Dans le vent du siècle, il a probablement prêté une oreille complaisante aux recommandations des physiocrates et défriché pour son compte sans trop s'interroger sur les questions de légitimité puisque lors de la vente de la ferme de Malhôtel, devenue bien national, celle-ci fait l'objet de réserves de la part de la municipalité qui revendique une partie des savarts dont elle dit avoir été spoliée. Il est enfin ouvert aux idées nouvelles et s'est suffisamment engagé dans le processus éruptif du printemps 89 pour avoir été député aux États généraux. Certes il fut un constituant discret qui ne laisse de trace ni au Moniteur ni dans le vote des lois. Il signe néanmoins le serment du Jeu de Paume et son niveau d'instruction ne doit pas être négligeable puisqu'il participe au Comité de Rédaction et à celui d'Agriculture et Commerce de l'Assemblée où il est dénommé Bailly du Vermandois sans doute pour le différencier du maire de Paris. Pour avoir côtoyé au Comité de Rédaction des grands noms de la Révolution comme Pétion, Barnave, Le Chapelier ou encore Buzot, on peut présumer qu'il a été témoin privilégié des événements. Effacé à Paris, il est en revanche très présent dans l'Aisne. Il saisit sans état d'âme l'opportunité de la vente des biens des religieux de Nogent-sous-Coucy, prouvant qu'il sait choisir son camp et qu'il a le sens de ses intérêts. Ainsi, le 17 mars 1791, il achète la ferme de Limonval, 280 arpents au terroir de Crécy-au-Mont et rachète en troisième main à une certaine veuve Huguet, le 4 août suivant, la ferme de Malhôtel qu'il tenait jusque-là en location ${ }^{26}$. Si l'on ajoute qu'un mois plus tard, le 8 septembre, le couple Bailly prend à bail pour 9 ans la ferme de Bonne Maison ${ }^{27}, 228$ arpents de terres labourables et 5 faux de prés, ci-devant propriété, elle aussi, des religieux de Nogent, achetée le 24 mars 1791 et rachetée en deuxième main, le 25 juillet de la même année, par la même veuve Huguet $^{28}$, on n'affirmera pas saisir toutes les subtilités relationnelles et les complicités qui accompagnent ces opérations, mais on comprendra très vite à qui profite alors la Révolution. L'exemple concret de Malhôtel éclairera l'ampleur des avantages d'une telle transaction pour le fermier : aux termes de son dernier bail du 4 avril 1785, Bailly versait chaque année la somme de 1593 livres ; il servira pour son achat, désormais, des annuités de 4758 livres pendant douze ans soit, avec les intérêts, trois fois le montant de la location annuelle. Mais la dépréciation puis l'effondrement de l'assignat vont considérablement embellir les conditions de cette transaction déjà exceptionnellement favorable. On peut en effet considérer que Bailly n'a pas versé plus de quatre ou cinq annuités en monnaie à valeur constante.

Tout aurait pu en rester là sans la mauvaise humeur d'Étienne Legris, fermier de Limonval et de Bonne Maison qui, par suite de l'achat de Limonval puis de la location de 
Bonne Maison par Bailly, se trouve sous la menace d'une exclusion à brève échéance. Toujours est-il que deux ans après l'adjudication de ces grandes fermes, à deux reprises, le 7 mai et le 22 juin 1793, il fait citer Bailly et plusieurs témoins au Bureau de paix du district en vue d'une conciliation. Au cours de ces audiences, on apprend que la vente de Limonval du 17 mars 1791 a été annulée en juillet de la même année par décision du directoire départemental tandis que le tribunal du district la confirme au contraire le 20 mars 1793. Legris conteste également la validité de la vente de Bonne Maison en déclarant qu'il a l'intention d'interjeter appel d'une sentence de confirmation rendue par le tribunal de district le 6 mai 1793. Naturellement Bailly et le procureur de la veuve Huguet soutiennent le bien jugé de ces sentences qui vont dans leur sens.

11 Heureusement le registre des délibérations du directoire départemental ${ }^{29}$ apporte quelques lueurs sur cette affaire à rebondissements qui commence le 1er juillet 1791 lorsque Osselin, notaire à Soissons et procureur de Legris, demande l'annulation de l'adjudication du 17 mars sous prétexte que l'affiche annonçant la vente indiquait un montant de bail de 3530 livres - somme effectivement consignée au contrat notarial officiel - alors que Legris avait déposé sur le bureau du district une contre-lettre attestant qu'il en payait mille de plus. Cette publicité mensongère, argumentait Osselin, avait été de nature à peser sur la mise à prix et les enchères. On admettra que le moyen est assez chicanier et que son caractère malhonnête ne saute pas aux yeux. Le district a beau jeu de répondre que les conditions du bail ne servent qu'à établir une mise à prix n'entravant en rien le feu des enchères qui s'ensuit. La vente a effectivement donné lieu à une belle bataille entre hommes de loi de Chauny, Coucy, Paris et atteint près du double de la mise à prix. On a même vu enchérir Favereau, huissier à Chauny dont Legris est le client, au point qu'on peut se demander s'il ne montait pas sur ses ordres... Le directoire du département n'est pas loin, semble-t-il, de partager ce sentiment, mais il a été choqué par les prétentions de Bailly qui, dans son mémoire en réponse, ne cache pas ses intentions de faire casser pour illégalité le bail de son adversaire ; aussi décidet-il de demander conseil au Comité d'Aliénation de l'Assemblée nationale qui propose, lui, l'annulation de la vente et l'organisation d'une nouvelle adjudication. Bailly ne bénéficie donc pas de la moindre bienveillance de la part de ses collègues, c'est le moins qu'on puisse dire! Le directoire du district, invité à s'exécuter mais très mécontent d'avoir été mis en suspicion temporise, semble-t-il, puisqu'il faut attendre huit mois avant que le département, le 29 mars 1792, ne revienne à la charge en demandant une nouvelle adjudication le 5 avril. Mais le 1er avril, Bailly, arguant de n'avoir été prévenu de la nouvelle adjudication que le 31 mars, demande le délai de trois mois prévu dans ce cas par la loi. Accordé. Enfin, le 4 août, Legris manifeste son impatience et demande une clarification de la situation avant d'entreprendre des travaux dans la ferme. Il lui est rétorqué, qu'avant de s'exécuter, il devra justifier de son bail général et de son sousbail. Malheureusement pour lui, la Révolution qui abat le trône le 10 Août ajourne à nouveau ses espoirs : les nouvelles élections envoient Saint-Just siéger à la Convention et ses amis, notamment Thuillier, au nouveau directoire du district, plus fondé que jamais à ne pas céder aux injonctions de l'autorité supérieure. Bien que nous manquions de sources pour cette année-là, il est facile d'imaginer que le 20 mars 1793 le district est en position de force pour trancher à nouveau en faveur de Bailly. Pas en dernier ressort, bien sûr, voilà pourquoi Bailly croit bon de solliciter l'appui de SaintJust par l'intermédiaire de Garot lorsque Legris relance le débat en s'adressant au Bureau de paix à la fin du printemps. 

lettre directement liée à cette affaire. Comme Saint-Just n'a séjourné en tant qu'élu à Paris que pendant deux mois de juillet, ceux de 1793 et 1794, que pour 1794 de surcroît il aurait écrit messidor, c'est sans aucun doute celui de 1793 qu'il faut retenir. Le conventionnel peut à juste titre déplorer la rareté de ses loisirs puisqu'il écrit le jour même où il prononce le fameux rapport sur les Trente-deux... ! démunis, des ignorants et des impécunieux, qui, pour certains, ne sont pas même citoyens actifs ${ }^{30}$. On ne l'a jamais vu au cours de la période blérancourtoise défendre Bailly bien que celui-ci soit un habitué des tribunaux. Il s'y fait alors représenter par Hottin. Il est en effet insolite de voir l'inspirateur des décrets de ventôse, promoteur, comme Robespierre, de la petite propriété, affirmer son «sincère attachement " à un notable nanti comme Bailly. A priori la compassion irait plutôt à sa victime, mais il faut en toute équité préciser que Legris n'est pas un prolétaire de la terre. Il s'est installé récemment dans ce bailliage, venu de la région plus méridionale d'Oulchy-le-Château où il paye en 1789 comme «laboureur" une contribution de 789 livres $^{31}$. C'est moins que Bailly, mais plus que Lemoine qui en paye 707 pour les 700 arpents de la ferme de Loire. Ce sont en fait des individus de même milieu qui occupent la crête du tiers état, ce qui ne veut pas dire qu'ils aient les mêmes convictions. Rien dans ce que nous savons de Legris n'indique qu'il s'implique dans la Révolution, au contraire. Dès qu'il a notification de l'annulation de la vente de Limonval par les autorités départementales, il s'empresse, comme au bon vieux temps, d'en payer les fermages à Augustin Roux cidevant fermier général des revenus de la ci-devant mense abbatiale de Nogent! On le retrouve par ailleurs dans une chicane mineure, toujours contre Bailly, comme fondé de pouvoir d'une dame de Suzy, veuve d'un lieutenant général des armées du roi ${ }^{32}$. Par idéal, par intérêt ou les deux, ses préférences vont manifestement à l'Ancien Régime. Bailly, comme on l'a vu, porte au contraire ses regards vers l'avenir. Élu aux États généraux, député à la Constituante, expert départemental en l'an IV et en l'an $\mathrm{V}^{33}$ dans l'estimation des biens des émigrés et du clergé, il termine sa vie conseiller général de son canton sous l'Empire. Il traverse ainsi quinze années de gros temps en passant du statut de locataire à celui de propriétaire. On ne sait si Saint-Just - qui se place prudemment dans la continuelle obligation de devoir rendre compte de sa mission - est effectivement intervenu en sa faveur ni comment s'est terminé ce feuilleton, mais le 19 novembre 1812 à une heure du matin, Charles Maximin Bailly décède "en sa maison de Limonval ». Quant à Legris, il n'apparait pas dans le dénombrement des habitants de Crécy-au-Mont en l'an IV.

Cette correspondance présente l'avantage de mieux délimiter les contours de ce qu'on peut appeler un réseau Saint-Just dont les motivations sont constituées à la fois de convictions et de désirs de promotions mais qui ne manque pas de fidélité. Dans ce département modéré dont tous les parlementaires sauf Saint-Just ont protesté solennellement contre l'arrestation des députés girondins, il est pittoresque de constater que deux villages ont envoyé à la Convention une adresse d'adhésion «aux journées du 31 mai, 1er et 2 juin »: Crécy-au-Mont et Coucy-le-Château ${ }^{34}$ ! Ce réseau inclut aussi des fermiers, en symbiose avec leurs notaires, qui sont souvent grands bénéficiaires des décisions législatives de 1790 , du moins quand ils ont les moyens d'acheter. Ils doivent en effet compter avec les rivalités qui les divisent et la concurrence de la bourgeoisie locale ou parisienne. Le cas de la plus belle ferme, celle 
de Loire, est exemplaire: enchérissent les fermiers les plus huppés de ce milieu endogame, les Lemoine, les Flobert, les Ferté (J.-B. Lemoine tout comme son père Roger, tuteur de Saint-Just, avaient épousé des Ferté) ; Bailly lui aussi enchérit ; mais tous doivent céder devant un homme de loi venu de Paris, acquéreur pour le compte d'un bourgeois nommé Lesage.

Les précisions de la seconde lettre permettent aussi de nuancer les idées économiques de Saint-Just et à travers lui celles des Robespierristes. Sa politique du lopin de terre aux démunis doit être considérée comme la volonté de leur donner une sorte de revenu minimum de subsistance et une garantie de dignité ; elle n'est pas conçue pour être généralisée. Il se montre trop imprégné de son expérience picarde et trop avisé dans ses discours économiques pour penser que le morcellement soit une panacée. On veut bien admettre par ailleurs que, comme tout député, il soit harcelé de sollicitations dont certaines sont peu recevables ou ambiguës, mais on ne peut croire qu'il ait transgressé ses convictions et ses principes pour ne pas mécontenter un soutien local ou pour faire plaisir à Garot, lui qui écrivait à sa sœur : « [...] ce que tu me demandes est contraire à la loi, il m'est impossible de te l'accorder ». Il accepte, du moins au début de l'été 1793, la réalité de ces grandes unités désormais affranchies de leurs rentes ecclésiastiques qui nourrissent Paris et ravitaillent les armées. Il n'est probablement pas le seul ni le dernier à être troublé par les éléments de contradiction qui viennent tourmenter tous ceux qui souhaitent conjuguer égalité et production. Faut-il faire un rapprochement avec cette curieuse note griffonnée et soulignée dans le carnet retrouvé sur lui le 9 Thermidor: «Ne pas admettre le partage des propriétés mais le partage des fermages »?

\section{NOTES}

1.Elle a été acquise par l'Association pour la sauvegarde de la maison de Saint-Just à Blérancourt (Aisne).

2.Orthographe et ponctuation corrigées ; mise entre crochets de mots manquants.

3.Huissier à Coucy-le-Château.

4.Saint-Just a rayé " préjudiciait », mais omis de rayer également " décidait ni ne ». 5.Bernard Vinot, Saint-Just, Paris Fayard, 1985, p. 24.

6.A.D. Aisne : C 195 et C 1021, affaire Doualle.

7.Bibliothèque de Soissons, fonds Perin, cahier de doléances de Juvigny.

8.Il anima son étude notariale jusqu'à sa mort en 1817 et son fils aîné lui succéda.

9.Secrétaire adjoint de la municipalité de Coucy en 1790 et inspecteur général des transports militaires sous les ordres de Daubigny en thermidor II.

10.A. KuPIEC et M. ABeNSOUR, Saint-Just, ceuvres complètes, Paris, Gallimard 2004, p. 1157, mais mal lu et transcrit Haulain.

11.En ventôse an III, Daubigny, accusé de robespierrisme, est incarcéré à la prison de Port-Libre d'où il rédige un « Précis justificatif et historique... ». Il y dénonce la noirceur de l'âme de Saint-Just dont il affirme détenir la preuve qu'il divulguera, assure-t-il, le moment venu. La lettre que lui a adressé Saint-Just dans un moment de 
dépression aurait bien pu servir de monnaie d'échange, ce qui expliquerait qu'elle n'apparaisse pas dans la première édition du Rapport.

12.Bernard VINOT, «La Révolution au village avec Saint-Just d'après le registre des délibérations communales de Blérancourt ", AHRF, 2004, n¹, p. 104.

13.La destruction des archives du bailliage pour cette période ne permet pas d'en connaître l'issue.

14.Registre des délibérations communales, $2 \mathrm{e}$ cahier, $15 \mathrm{e}$ feuillet, verso.

15.Elle faisait partie de la collection de l'avocat et député du Vermandois J-B Laurent Devisme (1749-1830), elle est conservée à la bibliothèque sous la cote $26 \mathrm{CA} 37 \mathrm{~N}$.

16.A. KuPIEC, op. cit., p. 1168.

17.Ce nom surchargé est de lecture difficile. Transcription approximative.

18.Il s'agit sans aucun doute de l'attachement de Saint-Just à Bailly et non l'inverse comme pourrait le laisser croire la transcription de A. KUPIEC, op. cit., p. 1168.

19.Ibid., p. 1154-1156.

20.Bernard Vinot, Saint-Just, op. cit., p. 84 et 103.

21.Cousin dont le père fut tuteur de Saint-Just orphelin.

22.A.D. Aisne, Etat-civil de Coucy-le-Château.

23.Gros village proche de Coucy implanté sur le riche plateau du Soissonnais.

24.L'arpent, le jaloi et la faux valent respectivement environ 42 , 35 et 45 ares.

25.A.D. Aisne, C 581.

26.Ibid., Q 432, nº8 et Q 433, nº137.

27.Ibid., $261 \mathrm{E} 104$.

28.Achetée en première main par un boucher de Chauny insolvable, cette ferme avait été revendue avec l'autorisation empressée du directoire du département ( $Q 411$, séance du 26 juillet 1791).

29.A.D. Aisne : Q412 et 413 aux dates indiquées.

30.Bernard VINOT, op. cit., p. 129-130.

31.A.D. Aisne, C 584, à la paroisse de Rozoy-les-Oulchy, aujourd'hui Grand Rozoy.

32.Ibid., L 2670, 21 avril 1792.

33.Ibid., Q 103, 104, 105, 109, 119.

34.Archives parlementaires, t. LXX, p. 20.

\section{AUTEUR}

\section{BERNARD VINOT}

7 rue du général Leclerc, 02300 Chauny, bernard.vinot@tele2.fr 